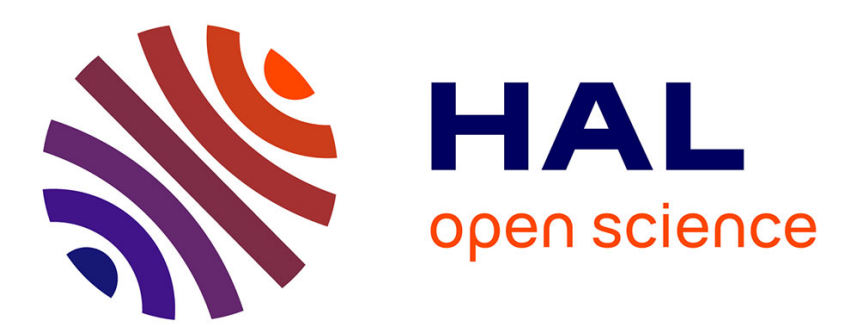

\title{
Blind subspace-based eigenstructure identification under nonstationary excitation using moving sensors
}

Laurent Mevel, Albert Benveniste, Michèle Basseville, Maurice Goursat

\section{To cite this version:}

Laurent Mevel, Albert Benveniste, Michèle Basseville, Maurice Goursat. Blind subspace-based eigenstructure identification under nonstationary excitation using moving sensors. [Research Report] RR4024, INRIA. 2000. inria-00072616

\section{HAL Id: inria-00072616 https://hal.inria.fr/inria-00072616}

Submitted on 24 May 2006

HAL is a multi-disciplinary open access archive for the deposit and dissemination of scientific research documents, whether they are published or not. The documents may come from teaching and research institutions in France or abroad, or from public or private research centers.
L'archive ouverte pluridisciplinaire HAL, est destinée au dépôt et à la diffusion de documents scientifiques de niveau recherche, publiés ou non, émanant des établissements d'enseignement et de recherche français ou étrangers, des laboratoires publics ou privés. 


\section{Blind subspace-based eigenstructure identification under nonstationary excitation using moving sensors}

Laurent Mevel, Albert Benveniste, Michèle Basseville, Maurice Goursat

\section{$\mathbf{N}^{\circ} 4024$}

Octobre 2000

THÈME 4

\section{apport}

derecherche 



\title{
RINRIA
}

\section{Blind subspace-based eigenstructure identification under nonstationary excitation using moving sensors}

\author{
Laurent Mevel, Albert Benveniste*, Michèle Basseville ${ }^{\dagger}$, Maurice Goursat ${ }^{\ddagger}$ \\ Thème 4 - Simulation et optimisation \\ de systèmes complexes \\ Projet Sigma2
}

Rapport de recherche $\mathrm{n}^{\circ} 4024$ - Octobre $2000-25$ pages

\begin{abstract}
Subspace identification algorithms have proven efficient for performing output-only identification of the eigenstructure of a linear MIMO system subject to uncontrolled, unmeasured, and nonstationary excitation. Such a problem arises in mechanical engineering, for modal analysis of vibrating structures and machines. A common practice there is to collect data from varying sensor locations, using both fixed and moving sensors, in order to mimic the availability of a larger set of sensors. The purpose of this paper is to investigate how subspace-based identification can be adapted to handle such a situation, to prove its consistency under nonstationary excitation, and to report on a real application example.
\end{abstract}

Key-words: Blind eigenstructure identification, nonstationary excitation, subspace algorithms, moving sensors, vibration analysis.

(Résumé : tsvp)

This work has been carried out within the framework of Eureka project 1562 Sinopsys (model based Structural monitoring using IN-OPeration SYStem identification), coordinated by LMS, Leuven, Belgium.

\footnotetext{
* IRISA/INRIA - Laurent.Mevel, benveniste@irisa.fr.

$\dagger$ IRISA/CNRS - basseville@irisa.fr.

$\ddagger$ INRIA - Maurice.Goursat@inria.fr.
} 


\section{Méthode sous-espace pour l'identification aveugle de la structure propre d'un système linéaire sous excitation non-stationnaire en utilisant des capteurs mobiles.}

Résumé : Les algorithmes d'identification par sous-espaces ont fait leurs preuves pour l'identification aveugle de la structure propre d'un système linéaire soumis à une excitation non contrôlée, non mesurée et non-stationnaire. Ce type de problème intervient en génie mécanique, pour l'analyse modale de structures ou machines en vibrations. Il est d'usage courant, dans ce domaine, de collecter des données en provenance de capteurs - certains fixes, d'autres mobiles - placés à différents endroits, afin d'imiter une situation où un nombre de capteurs beaucoup plus important serait disponible. Dans cet article, on étudie comment adapter l'identification par sous-espaces à une telle situation, de démontrer sa consistance sous excitation non-stationnaire, et de présenter des résultats expérimentaux obtenus sur données réelles.

Ce travail a été effectué dans le cadre du projet Eureka no 1562 Sinopsys (Model based Structural monitoring using in-operation system identification), qui est coordonné par la société Lms, Leuven, Belgique.

Mots-clé : Identification aveugle de structure propre, excitation non-stationnaire, algorithmes de type sous-espace, capteurs mobiles, analyse des vibrations. 


\section{Introduction}

Subspace-based linear system identification methods [7, 12, 13] have proven efficient for the identification of the eigenstructure of a linear multi-variable system. An important instance of this problem is structural vibration analysis, based on measurements from accelerometers or strain gauges, where the key issue is to identify vibrating characteristics (modes and modal shapes) of mechanical structures subject to uncontrolled, unmeasured and nonstationary excitation. Typical examples are offshore structures subject to swell, buildings subject to wind or earthquake, bridges, dams, wings subject to flutter in flight, and turbines subject to steam turbulence, friction in bearings, and imperfect balancing. A relevant approach to structural analysis has been shown to rely on the modeling of modes and modal shapes through state space representations [10], and the use of output-only and covariance-driven eigenstructure identification methods $[6,1]$.

A common practice in vibration analysis is to collect data from varying sensor locations, using both fixed and moving sensors, in order to mimic the availability of a (much) larger set of sensors. Several successive data sets are recorded, with sensors at different locations on the structure. Some of the sensors, called the reference sensors, are kept fixed, while the others are moved. This setup, usually referred to as polyreference setup [5] and typically based on about ten sensors, can mimic a situation in which hundreds of sensors are available.

Processing polyreference data for structural analysis is achieved today by performing eigenstructure identification for each record separately, and then merging the results obtained for records corresponding to different sensor pools. However, pole matching may be not easy in practice, and thus the result of eigenvector gluing may not be consistent. Therefore the question arises to perform eigenstructure identification by merging the data of the successive records, and processing them globally, instead of merging the identification results.

The purpose of this paper is to investigate how subspace-based identification methods can be adapted to handle a polyreference setup, in the presence of an unmeasured and nonstationary excitation. In section 2 , we briefly review output-only covariance-driven subspace identification methods. The mathematical modeling of the polyreference setup is stated in section 3. A first merge of the data is proposed for the stationary excitation case, and shown to fail in the nonstationary one. In section 4 , a covariance normalization prior to merge is proposed for the simplified case of record-dependent excitation, and its implementation is given. The fully nonstationary case, in which the excitation is nonstationary within the records, is addressed in section 5 , where a consistency result is proven. Numerical results obtained on a real 
application example are reported in section 6 . Finally, some conclusions are drawn in section 7 .

\section{Output-only covariance-driven subspace identification}

We consider linear multi-variable systems described by a discrete-time state space model

$$
\left\{\begin{aligned}
X_{k+1} & =F X_{k}+V_{k+1} \\
Y_{k} & =H X_{k}
\end{aligned}\right.
$$

where state $X$ and observed output $Y$ have dimensions $n$ and $r$, respectively, and where the state noise process $\left(V_{k}\right)_{k}$ is an unmeasured Gaussian white noise sequence with zero mean and, in the stationary case, constant covariance matrix $Q$ :

$$
\mathbf{E} V_{k} V_{k^{\prime}}^{T} \stackrel{\text { def }}{=} Q \delta\left(k-k^{\prime}\right)
$$

where $\mathbf{E}($.$) denotes the expectation operator. The whiteness assumption on the state$ noise and the absence of measurement noise in (1) are further discussed in section 6 . Let the pairs $\left(\lambda, \Phi_{\lambda}\right)$ be the eigenvalues and eigenvectors of the state transition matrix

$F$, and define $\varphi_{\lambda} \stackrel{\text { def }}{=} H \Phi_{\lambda}$. We assume that the system has no multiple eigenvalues, and thus that the $\lambda$ 's and $\varphi_{\lambda}$ 's are pairwise complex conjugate. In particular, 0 is not an eigenvalue of state transition matrix $F$. The collection of pairs $\left(\lambda, \varphi_{\lambda}\right)$ form a canonical parameterization of the pole part of system (1), referred to as the system eigenstructure. A canonical parameterization is a parameterization which is invariant w.r.t. changes in the state basis.

Processing output covariance matrices is of interest for very large data sets. The difference between the covariance-driven form of subspace algorithms which we describe now and the usual data-driven form is minor, at least for eigenstructure identification $[12,8]$. Covariance-driven subspace identification of the eigenstructure $\left(\lambda, \varphi_{\lambda}\right)$ 's is based on the following steps. Let

$$
R_{i} \stackrel{\text { def }}{=} \mathbf{E} Y_{k} Y_{k-i}^{T}
$$

and

$$
\mathcal{H}_{p+1, q} \stackrel{\text { def }}{=}\left(\begin{array}{cccc}
R_{0} & R_{1} & \vdots & R_{q-1} \\
R_{1} & R_{2} & \vdots & R_{q} \\
\vdots & \vdots & \vdots & \vdots \\
R_{p} & R_{p+1} & \vdots & R_{p+q-1}
\end{array}\right) \stackrel{\text { def }}{=} \operatorname{Hank}\left(R_{i}\right)
$$


be the theoretical output-covariance and Hankel matrices, respectively. Introducing the cross-covariance between the state and the observed outputs:

$$
G \stackrel{\text { def }}{=} \mathbf{E} X_{k} Y_{k}^{T}
$$

direct computations of the $R_{i}$ 's from the model equations lead to

$$
R_{i}=H F^{i} G
$$

and to the well known [11] factorization

$$
\mathcal{H}_{p+1, q}=\mathcal{O}_{p+1}(H, F) \mathcal{C}_{q}(F, G)
$$

where

$$
\mathcal{O}_{p}(H, F) \stackrel{\text { def }}{=}\left(\begin{array}{l}
H \\
H F \\
\vdots \\
H F^{p-1}
\end{array}\right)
$$

and

$$
\mathcal{C}_{q}(F, G) \stackrel{\text { def }}{=}\left(G F G \cdots F^{q-1} G\right)
$$

are the observability and controllability matrices, respectively. In the sequel, we often drop the orders of the system matrices.

The observation matrix $H$ is then found in the first block-row of the observability matrix $\mathcal{O}$, whereas exploiting the shift invariance property of $\mathcal{O}$ provides us with the state-transition matrix $F$, from which the eigenstructure $\left(\lambda, \varphi_{\lambda}\right)$ results. The key feature in factorization (4) which will be elaborated on, is that the left factor $\mathcal{O}$ only depends on the pair $(H, F)$, and thus on the eigenstructure of system (1), whereas the excitation $V_{k}$ only affects the right factor $\mathcal{C}$ through the cross-covariance matrix $G$.

The actual implementation of this subspace algorithm has the empirical covariances

$$
\widehat{R}_{i}=\frac{1}{N} \sum_{k=1}^{N} Y_{k} Y_{k-i}^{T}
$$

substituted for $R_{i}$ in $\mathcal{H}_{p+1, q}$, yielding the empirical Hankel matrix $\widehat{\mathcal{H}}_{p+1, q}$. How to select the number of lags $(p+q)$ and thus the size of $\widehat{\mathcal{H}}_{p+1, q}$ is discussed in section 6 . The SVD of $\widehat{\mathcal{H}}_{p+1, q}$ and its truncation at the desired model order yield, in the left factor, an estimate $\widehat{\mathcal{O}}$ for the observability matrix $\mathcal{O}$, from which $(\widehat{H}, \widehat{F})$ and $\left(\widehat{\lambda}, \widehat{\varphi}_{\lambda}\right)$ are recovered as sketched above.

$\mathrm{RR} \mathrm{n}^{\circ} 4024$ 


\section{Polyreference subspace identification: stationary exci- tation}

Instead of a single record for the output $\left(Y_{k}\right)$ of system (1), we now have $J$ records

$$
\underbrace{\left(\begin{array}{c}
Y_{k}^{(0,1)} \\
Y_{k}^{(1)}
\end{array}\right)}_{\text {Record } 1} \underbrace{\left(\begin{array}{c}
Y_{k}^{(0,2)} \\
Y_{k}^{(2)}
\end{array}\right)}_{\text {Record 2 }} \cdots \underbrace{\left(\begin{array}{c}
Y_{k}^{(0, J)} \\
Y_{k}^{(J)}
\end{array}\right)}_{\text {Record } J}
$$

collected successively. Each record $j$ contains data $Y_{k}^{(0, j)}$ from a fixed reference sensor pool, and data $Y_{k}^{(j)}$ from a moving sensor pool. The number of sensors may be different in the fixed and the moving pools, and thus in each record $j$, the measurement vectors $Y_{k}^{(0, j)}$ and $Y_{k}^{(j)}$ may have different dimensions. This is known under the name of polyreference setup [5].

To each record $j(1 \leq j \leq J)$ corresponds a state-space realization in the form

$$
\left\{\begin{aligned}
X_{k+1}^{(j)} & =F X_{k}^{(j)}+V_{k+1}^{(j)} & & \\
Y_{k}^{(0, j)} & =H_{0} X_{k}^{(j)} & & (\text { reference pool) } \\
Y_{k}^{(j)} & =H_{j} X_{k}^{(j)} & & \text { (sensor pool n } \left.{ }^{o} j\right)
\end{aligned}\right.
$$

with a single state transition matrix $F$ - since the same system is being observed, a fixed observation matrix $H_{0}$ for the fixed sensor pool, and a specific observation matrix $H_{j}$ corresponding to location $j$ of the moving sensor pool.

The problem is to find how to adapt the output-only covariance-driven subspace algorithm of section 2 in order to identify the eigenstructure of $F$ in (7), on the basis of the measurements (6) which we should merge somehow for this purpose. At this point, two points should be stressed. First, the cornerstone in section 2 is factorization (3) which, of course, holds for each of the $J$ records with a different left factor $H$ and a different right factor $G$. In particular, even though we do have access to empirical estimates of the covariances $\mathbf{E} Y_{k}^{(j)} Y_{k-i}^{(j)^{T}}$ of the moving sensor pool, they are of poor help because their factorizations (3) show up a record-dependent state-output correlation $G$ since the sensors location change. Second, we have no access to cross-record covariances $\mathbf{E} Y_{k}^{\left(j^{\prime}\right)} Y_{k-i}^{(j)^{T}}$ with $j \neq j^{\prime}$, since sensor pools $j$ and $j^{\prime}$ do not record data simultaneously. Thus, we cannot stack all the data sets in a unique vector, otherwise we would have to handle incomplete covariance matrices.

A consequence of those two facts, in the perspective of building a Hankel matrix enjoying a factorization property, is to concentrate on the covariances involving the 
reference sensors. From now on, we thus focus on the two following families of covariances

$$
R_{i}^{0, j} \stackrel{\text { def }}{=} \mathbf{E} Y_{k}^{(0, j)} Y_{k-i}^{(0, j)^{T}}, \quad R_{i}^{j} \stackrel{\text { def }}{=} \mathbf{E} Y_{k}^{(j)} Y_{k-i}^{(0, j)^{T}}
$$

of which we can compute empirical estimates, for lags $i \geq 0$.

A first merge of the data is now proposed for the stationary input excitation case. It is shown to fail in the nonstationary case, even under the simplifying assumption of stationary excitation within the records.

Constant excitation covariance matrix In the stationary case, the excitation covariance matrix does not depend on record $j$ :

$$
\mathbf{E} V_{k}^{(j)} V_{k^{\prime}}^{(j)^{T}}=Q \delta\left(k-k^{\prime}\right)
$$

and the cross-covariance between the state and the fixed sensors output

$$
G \stackrel{\text { def }}{=} \mathbf{E} X_{k}^{(j)} Y_{k}^{(0, j)^{T}}
$$

does not depend on $j$ either. Therefore, for all the records $j=1, \ldots, J$ and lags $i \geq 0$, the covariances in (8) factorize, with a constant right factor, as

$$
\begin{aligned}
R_{i}^{0, j} & =H_{0} F^{i} G \stackrel{\text { def }}{=} R_{i}^{0} \\
R_{i}^{j} & =H_{j} F^{i} G
\end{aligned}
$$

Consequently, for each lag $i \geq 0$, we can stack the $R_{i}^{j}$ 's into a block-column vector

$$
R_{i}^{\pi} \stackrel{\text { def }}{=}\left(\begin{array}{c}
R_{i}^{0} \\
R_{i}^{1} \\
\vdots \\
R_{i}^{J}
\end{array}\right)
$$

which factorizes as

$$
R_{i}^{\pi}=H F^{i} G
$$

where $H$ is the block-column of observation matrices

$$
H \stackrel{\text { def }}{=}\left(\begin{array}{c}
H_{0} \\
H_{1} \\
\vdots \\
H_{J}
\end{array}\right)
$$

$\mathrm{RR} \mathrm{n}^{\circ} 4024$ 
Therefore, the Hankel matrix filled with those column-stacked covariances, which has $(J+1)$ times as many rows as columns, factorizes as

$$
\mathcal{H}^{\pi} \stackrel{\text { def }}{=} \operatorname{Hank}\left(R_{i}^{\pi}\right)=\mathcal{O}(H, F) \mathcal{C}(F, G)
$$

and the algorithm described in section 2 for the standard case can be applied to $\mathcal{H}^{\pi}$. In other words, when the input excitation is stationary, we can proceed as if we had the full set of sensor data available altogether, instead of sensor pools with varying locations. In this case, direct application of the subspace algorithm to the column-stacked covariances (10) is a simple solution to the polyreference output-only eigenstructure identification problem.

Record-dependent excitation covariance matrix If we assume that the input excitation covariance matrix depends on the record index $j$ :

$$
\mathbf{E} V_{k}^{(j)} V_{k^{\prime}}^{(j)^{T}}=Q_{j} \delta\left(k-k^{\prime}\right)
$$

the cross-covariance matrix

$$
G_{j} \stackrel{\text { def }}{=} \mathbf{E}_{j} X_{k}^{(j)} Y_{k}^{(0, j)^{T}}
$$

also depends on $j$. Hence, for $j=1, \ldots, J$ and $i \geq 0$, factorizations (9) now write with a record-dependent $G$

$$
\begin{aligned}
R_{i}^{0, j} & =H_{0} F^{i} G_{j} \\
R_{i}^{j} & =H_{j} F^{i} G_{j}
\end{aligned}
$$

and vector $R_{i}^{\pi}$ of stacked covariances defined in (10) no longer factorizes as in (11). As a consequence, brute force application of subspace algorithm by stacking the $R_{i}^{j}$ 's is not an appropriate solution to the eigenstructure identification problem in the case of record-dependent input excitation noise. To circumvent this difficulty, the idea is to normalize the covariance matrices (14)-(15) to make them looking as if they were obtained with the same excitation.

\section{Nonstationary excitation: data normalization}

For finding the appropriate normalization, we perform different covariance stacking operations for the data from the reference and moving sensors, respectively. In what follows, we use the generic notations introduced in section 2 . 
Reference sensors Thanks to (14), all the Hankel matrices built on the data delivered by the reference sensors of the $J$ records factorize with the same left factor as

$$
\mathcal{H}_{0, j} \stackrel{\text { def }}{=} \operatorname{Hank}\left(R_{i}^{(0, j)}\right)=\mathcal{O}\left(H_{0}, F\right) \mathcal{C}\left(F, G_{j}\right)
$$

We can thus proceed as follows. Let

$$
R_{i}^{0} \stackrel{\text { def }}{=}\left(R_{i}^{0,1} R_{i}^{0,2} \ldots R_{i}^{0, J}\right)
$$

be the block-row vector of the entire set of reference data covariances. It factorizes as

$$
R_{i}^{0}=H_{0} F^{i} G, \quad \text { where } G \stackrel{\text { def }}{=}\left(G_{1} G_{2} \ldots G_{J}\right)
$$

Thus the Hankel matrix filled with those row-stacked covariances, which has $J$ times as many columns as rows, also factorizes as

$$
\mathcal{H}_{0} \stackrel{\text { def }}{=} \operatorname{Hank}\left(R_{i}^{0}\right)=\mathcal{O}\left(H_{0}, F\right) \mathcal{C}(F, G)
$$

Note that matrix $\mathcal{H}_{0}$ can be obtained by interleaving the block-columns of the $J$ Hankel matrices $\mathcal{H}_{0, j}$ in (16). Assume that pair $\left(H_{0}, F\right)$ is observable. Then, partitioning the right factor $\mathcal{C}$ of (18) in the same manner as $G$ in (17), we recover the right factors $\mathcal{C}\left(F, G_{j}\right)$ of (16) for all $j=1, \ldots, J$. Note that processing the reference data $Y_{k}^{(0, j)}$ altogether, on the basis of the single Hankel matrix factorization (18), is mandatory for making sure that we deal with the same state bases in (7).

Moving sensors Thanks to (15), the Hankel matrix built on the covariances of the data $Y_{k}^{(j)}$ from the moving sensor pool in record $j$ enjoys a record-dependent factorization

$$
\mathcal{H}_{j} \stackrel{\text { def }}{=} \operatorname{Hank}\left(R_{i}^{j}\right)=\mathcal{O}\left(H_{j}, F\right) \mathcal{C}\left(F, G_{j}\right)
$$

Assume that, for all $j$, the pair $\left(F, G_{j}\right)$ is controllable. This is a reasonable assumption, it just tells that, while the excitation may change, we need it to excite all the modes of the system, for each data set. Assume also that, up to a permutation on the record indexes, $\mathcal{C}\left(F, G_{1}\right)$ is the best conditioned controllability matrix among the $\mathcal{C}\left(F, G_{j}\right)$ 's. For all $j, j=1, \ldots, J$, we define the normalized matrices

$$
\overline{\mathcal{H}}_{j} \stackrel{\text { def }}{=} \mathcal{H}_{j}\left(\mathcal{C}^{T}\left(F, G_{j}\right)\left(\mathcal{C}\left(F, G_{j}\right) \mathcal{C}^{T}\left(F, G_{j}\right)\right)^{-1} \mathcal{C}\left(F, G_{1}\right)\right)
$$


We note that $\overline{\mathcal{H}}_{j}$ coincides with the Hankel matrix $\operatorname{Hank}\left(\bar{R}_{i}^{j}\right)$ built on covariances $\bar{R}_{i}^{j}$ which factorize as

$$
\bar{R}_{i}^{j}=H_{j} F^{i} G_{1}
$$

Therefore, we can now proceed as in the case of constant excitation covariance matrix. For each lag $i \geq 0$, we define the block-column stacked covariances

$$
\bar{R}_{i} \stackrel{\text { def }}{=}\left(\begin{array}{c}
\bar{R}_{i}^{0} \\
\bar{R}_{i}^{1} \\
\vdots \\
\bar{R}_{i}^{J}
\end{array}\right), \quad \text { where } \bar{R}_{i}^{0} \stackrel{\text { def }}{=} R_{i}^{0,1}=H_{0} F^{i} G_{1}
$$

Thanks to (21), those stacked covariances $\bar{R}_{i}$ behave as $R_{i}^{\pi}$ defined in (10): they factorize as

$$
\bar{R}_{i}=H F^{i} G_{1}
$$

with $H$ defined in (12). Therefore, the Hankel matrix filled with those columnstacked covariances, which has $(J+1)$ times as many rows as columns, factorizes as

$$
\overline{\mathcal{H}} \stackrel{\text { def }}{=} \operatorname{Hank}\left(\bar{R}_{i}\right)=\mathcal{O}(H, F) \mathcal{C}\left(F, G_{1}\right)
$$

Note that matrix $\overline{\mathcal{H}}$ can be obtained by interleaving the block-rows of

$$
\overline{\mathcal{H}}_{0} \stackrel{\text { def }}{=} \operatorname{Hank}\left(\bar{R}_{i}^{0}\right)=\mathcal{H}_{0,1}
$$

and of the $J$ matrices $\overline{\mathcal{H}}_{j}$ defined in (20). Thanks to (23), the algorithm given in section 2 can be applied to $\overline{\mathcal{H}}$.

Algorithm For implementing the above reasoning, we propose the following algorithm, where $J$ is the number of records and $\widehat{M}$ denotes an empirical estimate of matrix $M$.

- Build the $J$ Hankel matrices $\widehat{\mathcal{H}}_{0, j}=\operatorname{Hank}\left(\widehat{R}_{i}^{(0, j)}\right)$ from the reference sensor data.

- Build $\widehat{\mathcal{H}}_{0}$ by interleaving the block-columns of the $J$ matrices $\widehat{\mathcal{H}}_{0, j}$ 's $(j=$ $1, \ldots, J)$.

- SVD-decompose $\widehat{\mathcal{H}}_{0}$. Keep only the right factor $\mathcal{C}(F, G)$ in (18), and call it just $\mathcal{C}$. 
- Partition $\mathcal{C}$ into $\mathcal{C} \stackrel{\text { def }}{=}\left(\begin{array}{lllll}\mathcal{C}_{1} & \mathcal{C}_{2} & \ldots & \mathcal{C}_{J}\end{array}\right)$ as $G$ in (17). Assume $\mathcal{C}_{1}$ is the best conditioned.

- Compute the normalizing factors $\left(\mathcal{C}_{j}^{T}\left(\mathcal{C}_{j} \mathcal{C}_{j}^{T}\right)^{-1} \mathcal{C}_{1}\right)$.

- Build the $J$ Hankel matrices $\widehat{\mathcal{H}}_{j}=\operatorname{Hank}\left(\widehat{R}_{i}^{j}\right)$ from the moving sensor data.

- Compute the $J$ normalized matrices $\overline{\mathcal{H}}_{j} \stackrel{\text { def }}{=} \widehat{\mathcal{H}}_{j}\left(\mathcal{C}_{j}^{T}\left(\mathcal{C}_{j} \mathcal{C}_{j}^{T}\right)^{-1} \mathcal{C}_{1}\right)$. Define $\widehat{\widehat{\mathcal{H}}}_{0} \stackrel{\text { def }}{=} \widehat{\mathcal{H}}_{0,1}$.

- Build $\widehat{\mathcal{H}}$ by interleaving the block-rows of the $(J+1)$ matrices $\overline{\mathcal{H}}_{j}$ 's $(j=$ $0, \ldots, J)$.

- Apply the algorithm of section 2 to $\widehat{\overline{\mathcal{H}}}$.

The following comments are in order. First, the computational complexity of this algorithm does not depend on the number $J$ of records, since the smallest dimension of the two Hankel matrices $\widehat{\mathcal{H}}_{0}$ and $\widehat{\overline{\mathcal{H}}}$ to be decomposed depends only on the number of reference sensors. Second, the interleaving of the block-rows of the $\overline{\mathcal{H}}_{j}$ 's can be done even when the number of sensors in the moving pools is not constant: the measurement vectors $Y_{k}^{(j)}$ and $Y_{k}^{\left(j^{\prime}\right)}$ may have different dimensions. Third, we do not need to compute explicitly the $G_{j}$ matrices, since only the right factors $\mathcal{C}\left(F, G_{j}\right)$ in (19) are needed for computing $\widehat{\widehat{\mathcal{H}}}_{j}$ in (20).

\section{$5 \quad$ Nonstationary excitation within the records}

In section 4 , we have assumed stationary excitation within each record, with recorddependent covariance matrix. This is a reasonable approximation when the environmental conditions are slowly time-varying. But a more realistic assumption is that the excitation covariance matrix:

$$
\mathbf{E} V_{k}^{(j)} V_{k^{\prime}}^{(j)^{T}}=Q_{k} \delta\left(k-k^{\prime}\right)
$$

is time-varying within each record. Hence the output data of the polyreference setup (7) cannot be assumed stationary within each individual record, and we cannot apply the argument of section 4 . Nevertheless, the algorithm described in that section can still be applied with little modification, and can be proven consistent, as we show now using the results of [4].

$\mathrm{RR} \mathrm{n}^{\circ} 4024$ 
For $j=1, \ldots, J$, let $\mathcal{I}_{j}$ be the finite set of sample points in record $j$, let $K_{j} \stackrel{\text { def }}{=}\left|\mathcal{I}_{j}\right|$ be the number of samples, and define the following second order empirical moments

$$
\begin{aligned}
A_{j}\left(K_{j}\right) & \stackrel{\text { def }}{=} \frac{1}{2 K_{j}} \sum_{k \in \mathcal{I}_{j}}\left(\left\|Y_{k}^{(0, j)}\right\|^{2}+\left\|Y_{k}^{j}\right\|^{2}\right) \\
G_{j}\left(K_{j}\right) & \stackrel{\text { def }}{=} \frac{1}{A_{j}\left(K_{j}\right)} \sum_{k \in \mathcal{I}_{j}} X_{k}^{(j)} Y_{k}^{(0, j)^{T}} \\
R_{i}^{0, j}\left(K_{j}\right) & \stackrel{\text { def }}{=} \frac{1}{A_{j}\left(K_{j}\right)} \sum_{k \in \mathcal{I}_{j}} Y_{k}^{(0, j)} Y_{k-i}^{(0, j)^{T}} \\
R_{i}^{j}\left(K_{j}\right) & \stackrel{\text { def }}{=} \frac{1}{A_{j}\left(K_{j}\right)} \sum_{k \in \mathcal{I}_{j}} Y_{k}^{j} Y_{k-i}^{(0, j)}
\end{aligned}
$$

Consider the following assumptions, identical to conditions $\mathrm{C} 1$ and $\mathrm{C} 2$ of section II.A of [4].

\section{Assumptions 1}

C1: $\lim _{K_{j} \rightarrow+\infty} A_{j}\left(K_{j}\right)=+\infty$ with probability 1 .

C2: The excitation covariance matrix $Q_{k}$ is uniformly bounded from above, in $k$.

Note that condition $\mathbf{C 1}$ is a very mild request stating that, for each individual record $j$, the system is sufficiently excited. The following result is proved in the first step of the proof of theorem 1 in [4][Sec.III.A] :

Theorem 1 Under conditions C1-C2, we have

$$
\begin{aligned}
R_{i}^{0, j}\left(K_{j}\right) & =H_{0} F^{i} G_{j}\left(K_{j}\right)+o\left(K_{j}\right) \\
R_{i}^{j}\left(K_{j}\right) & =H_{j} F^{i} G_{j}\left(K_{j}\right)+o\left(K_{j}\right)
\end{aligned}
$$

where matrix o $(K)$ goes to zero when $K$ goes to infinity.

Theorem 1 expresses that (14)-(15) hold asymptotically. Consider now the following additional assumptions.

\section{Assumptions 2}

C3: For every $j$ and $K_{j}$ large, the $n$-th (in decreasing order) singular value of the controllability matrix $\mathcal{C}_{j}\left(K_{j}\right) \stackrel{\text { def }}{=} \mathcal{C}_{q}\left(F, G_{j}\left(K_{j}\right)\right)$ is asymptotically uniformly bounded from below with probability 1. 
C4: For every $j$, the pair $\left(H_{j}, F\right)$ is observable, that is : $\operatorname{rank} \mathcal{O}_{p}\left(H_{j}, F\right)=n$.

Condition C3, which coincides with condition C3 of [4][Sec.II.A], means that the pair $\left(F, G_{j}\left(K_{j}\right)\right)$ is asymptotically uniformly controllable for every $j$ and $K_{j}$ large.

Introduce the Hankel matrix

$$
\mathcal{H}^{j} \stackrel{\text { def }}{=} \operatorname{Hank}\left(R_{i}^{j}\left(K_{j}\right)\right)
$$

and the normalized matrix

$$
\overline{\mathcal{H}}^{j} \stackrel{\text { def }}{=} \mathcal{H}^{j}\left(\mathcal{C}_{j}^{T}\left(\mathcal{C}_{j} \mathcal{C}_{j}^{T}\right)^{-1} \mathcal{C}_{1}\right)
$$

where $\mathcal{C} \stackrel{\text { def }}{=}\left(\mathcal{C}_{1} \mathcal{C}_{2} \ldots \mathcal{C}_{J}\right)$ is the right factor of $\mathcal{H}^{0}$, and $\mathcal{H}^{0}$ is built by interleaving the block-columns of the $J$ matrices

$$
\mathcal{H}^{0, j} \stackrel{\text { def }}{=} \operatorname{Hank}\left(R_{i}^{0, j}\left(K_{j}\right)\right)
$$

Note that, as in (20), $\overline{\mathcal{H}}^{j}$ is an Hankel matrix built on covariances which we note $\bar{R}_{i}^{j}$. The following theorem holds.

Theorem 2 Under conditions $\mathbf{C 1 - C 4 , ~ t h o s e ~ c o v a r i a n c e s ~ e n j o y ~ t h e ~ a p p r o x i m a t e ~ f a c - ~}$ torization:

$$
\overline{R_{i}^{j}}=H_{j} F^{i} G_{1}\left(K_{1}\right)+o\left(K_{1}\right)
$$

where matrix $o\left(K_{1}\right)$ goes to zero when $K_{1}$ goes to infinity.

Proof For all the $K_{j}$ large, define

$$
\begin{gathered}
R_{i}^{j} \stackrel{\text { def }}{=} H_{j} F^{i} G_{j}\left(K_{j}\right) \\
\mathcal{H}_{j} \stackrel{\text { def }}{=} \operatorname{Hank}\left(R_{i}^{j}\right)
\end{gathered}
$$

and

$$
\overline{\mathcal{H}}_{j} \stackrel{\text { def }}{=} \operatorname{Hank}\left(H_{j} F^{i} G_{1}\left(K_{1}\right)\right)
$$

With these notations, theorem 1 writes

$$
R_{i}^{j}\left(K_{j}\right)=R_{i}^{j}+o\left(K_{j}\right)
$$


and we have the following relation for the Hankel matrices

$$
\overline{\mathcal{H}}_{j}=\mathcal{H}_{j}\left(\mathcal{C}_{j}^{T}\left(K_{j}\right)\left(\mathcal{C}_{j}\left(K_{j}\right) \mathcal{C}_{j}^{T}\left(K_{j}\right)\right)^{-1} \mathcal{C}_{1}\left(K_{1}\right)\right)
$$

For proving (26), it is sufficient to prove that, for all $j$ and for $K_{j}$ large, the following holds:

$$
\overline{\mathcal{H}}^{j}=\overline{\mathcal{H}}_{j}+o\left(\min _{j} K_{j}\right)
$$

To prove (28), we note that (25) and (27) result in

$$
\begin{gathered}
\overline{\mathcal{H}}^{j}-\overline{\mathcal{H}}_{j}=\mathcal{H}^{j} \underbrace{\left(\mathcal{C}_{j}^{T}\left(\mathcal{C}_{j} \mathcal{C}_{j}^{T}\right)^{-1} \mathcal{C}_{1}\right)}_{\mathcal{F}^{j}} \\
-\mathcal{H}_{j} \underbrace{\left(\mathcal{C}_{j}^{T}\left(K_{j}\right)\left(\mathcal{C}_{j}\left(K_{j}\right) \mathcal{C}_{j}^{T}\left(K_{j}\right)\right)^{-1} \mathcal{C}_{1}\left(K_{1}\right)\right)}_{\mathcal{F}_{j}}
\end{gathered}
$$

Thanks to condition C3, controllability matrices $\mathcal{C}_{1}$ and $\mathcal{C}_{j}$ are uniformly bounded from below, for $K_{1}$ and $K_{j}$ large, and matrix $\left(\mathcal{C}_{j} \mathcal{C}_{j}^{T}\right)^{-1}$ is uniformly bounded from above. This and theorem 1 together imply that, for $K_{j}$ large,

$$
\mathcal{H}^{j}=\mathcal{H}_{j}+o\left(K_{j}\right), \quad \mathcal{F}^{j}=\mathcal{F}_{j}+o\left(\min _{j} K_{j}\right)
$$

Equations (29) and (30) together prove (28). This concludes the proof of the theorem.

Theorems 1 and 2 ensure that the method of section 4 applies, provided that we use the $R_{i}^{0, j}\left(K_{j}\right)$ 's and $R_{i}^{j}\left(K_{j}\right)$ 's in $(24)$ as empirical estimates of the covariances. Furthermore, the following consistency can be proven, as in [4].

Theorem 3 Under conditions $\mathbf{C 1 - C 4 , ~ t h e ~ a l g o r i t h m ~ o f ~ s e c t i o n ~} 4$ is consistent: there exists a sequence of matrices $T$, with $T$ and $T^{-1}$ uniformly bounded, such that $T^{-1} \hat{F} T \rightarrow F, \quad \hat{H} \rightarrow H$ with probability 1 .

Since we have assumed that the system has no multiple eigenvalues and because the eigenstructure is a continuous function of the pair $(H, F)$, this theorem also ensures [4] the consistency of the eigenstructure estimate $\left(\hat{\lambda}, \widehat{\varphi}_{\lambda}\right)$.

\section{Structural vibration analysis example}

As mentioned in the introduction, structural vibration analysis of complex mechanical structures and rotating machines subject to ambient excitation is an important 
instance of eigenstructure identification. These systems are subject to fast and unmeasured variations in their environment, and thus the input excitation is typically turbulent in nature and nonstationary. For example, offshore structures are subject to the turbulent and highly time-varying action of the swell - shock effects due to fluid/structure interaction - which cannot be considered as measurable. The same holds true for wind and traffic on bridges, which result in unmeasured and nonstationary excitation. A different example concerns rotating machines such as huge alternators in electricity power plants [10].

In this section, we describe the application of the polyreference subspace identification method proposed in section 4 to structural vibration analysis. First, we introduce the modeling issues, and then we report some numerical results we have obtained on a real example.

\subsection{Modeling}

We assume that the behavior of the mechanical system can be described by a stationary linear dynamical system, and that, in the frequency range of interest, the input forces can be modeled as a nonstationary white noise. This results in the following matrix differential equation :

$$
\left\{\begin{aligned}
M \ddot{\mathcal{Z}}(t)+C \dot{\mathcal{Z}}(t)+K \mathcal{Z}(t) & =\nu(t) \\
Y(t) & =L \mathcal{Z}(t)
\end{aligned}\right.
$$

where $t$ denotes continuous time, $M, C, K$ are the mass, damping and stiffness matrices respectively, (high dimensional) vector $\mathcal{Z}$ collects the displacements or accelerations of the degrees of freedom of the structure; the external (non measured) force $\nu$ is modeled as a nonstationary white noise with time-varying covariance matrix $Q_{\nu}(t)$, measurements are collected in the (low dimensional) vector $Y$, and matrix $L$ indicates which components of the state vector are actually measured (where the sensors are located). Without loss of generality, we do not consider measurement noise, which can be encompassed in subspace algorithms by shifting the lags of the covariances used for filling the Hankel matrices [12, 2]. Sinusoidal or colored excitation noise can be encompassed as well [10]. How the presence and nature of excitation and measurement noises are handled in practice, when using an algorithm non encompassing them, is addressed in subsection 6.2.

The parameters $(M, C, K)$ cannot be recovered from measured outputs. Hence, identifiable parameters are introduced. These are the modes or eigen-frequencies $\mu$, and the modal shapes or eigenvectors $\psi_{\mu}$, solutions of:

$$
\left(M \mu^{2}+C \mu+K\right) \Psi_{\mu}=0, \quad \psi_{\mu}=L \Psi_{\mu}
$$

$\mathrm{RR} \mathrm{n}^{\circ} 4024$ 
Sampling model (31) at rate $1 / \tau$ yields the discrete time state space model (1), where the state and the output are :

$$
X_{k}=\left(\begin{array}{c}
\mathcal{Z}(k \tau) \\
\dot{\mathcal{Z}}(k \tau)
\end{array}\right), \quad Y_{k}=Y(k \tau)
$$

the state transition and observation matrices are :

$$
F=e^{\mathcal{L} \tau}, \mathcal{L}=\left(\begin{array}{cc}
0 & I \\
-M^{-1} K & -M^{-1} C
\end{array}\right), H=\left(\begin{array}{ll}
L & 0
\end{array}\right),
$$

and where state noise $V_{k+1}$ is zero-mean, white, with covariance matrix :

$$
Q_{k+1}=\int_{k \tau}^{(k+1) \tau} e^{\mathcal{L} s} \widetilde{Q}(s) e^{\mathcal{L}^{T} s} d s
$$

where

$$
\widetilde{Q}(s)=\left(\begin{array}{cc}
0 & 0 \\
0 & M^{-1} Q_{\nu}(s) M^{-T}
\end{array}\right)
$$

The modal parameters in (32) are equivalently found in the eigenstructure $\left(\lambda, \Phi_{\lambda}\right)$ of $F$ :

$$
e^{\tau \mu}=\lambda, \psi_{\mu}=\varphi_{\lambda} \stackrel{\text { def }}{=} H \Phi_{\lambda}
$$

Because of the structure of the state in (33), the eigenvectors are pairwise complex conjugate. They are real if proportional damping is assumed, that is if $C=\alpha M+\beta K$.

Therefore, vibration analysis can be stated as the problem of identifying the eigenstructure of the state transition matrix of a linear dynamic system with nonstationary state noise. State $X$ and observed output $Y$ have $\operatorname{dimensions} n=2 \operatorname{dim} \mathcal{Z}$ and $r$ respectively, with $r$ (much) smaller than $n$ in practice.

\subsection{Numerical results}

We now report some numerical results obtained with the polyreference subspace algorithm proposed in section 4. Before proceeding, some comments are in order on the practical use of the classical (monoreference and full covariance) subspace identification algorithm of section 2 . 
Some practical issues in subspace eigenstructure identification The selection of the model order, and thus of the size of the Hankel matrices on one hand, and the handling of the presence and nature of excitation and measurement noises on the other hand, are two major practical issues which we address now.

As stressed in $[10,3,8]$, when few sensors are available, a number of modes which is much smaller than the number of solutions of (32), but much larger than the number of sensors, can be identified. In order to extract the modes from the data, we have to apply the subspace method with an increasing SVD truncation order and to look for the relevant modes in the frequency band of interest. Since the subspace method yields a set of modes with both structural - from the structure and the excitation - and spurious mathematical modes, we have to derive a practical way to distinguish between the two types of modes. Hopefully, spurious modes tend to vary from one model order to the next. That is why usage suggests to plot the frequencies against SVD truncation order, in a so-called stabilization diagram [8]. Numerous experiments with the classical subspace method have shown that :

- It is of no practical help to select for $\mathcal{H}_{p+1, q}$ in (2) other values than $q=p+1$. The number of lags $2 p$ for the data covariances should be large (typically more than two hundreds in the experiments below),

- The number of modes should be overestimated, because modes tend to stabilize, and even to show up, for high orders. In other words, the SVD in the algorithm of section 2 should be truncated at an order much greater than the 'theoretical' order, that is $2 m / r-1$, where $m$ is the number of desired modes and $r$ is the number of sensors.

An excitation noise at a single constant frequency is recognized as a pole by the algorithm of section 2, designed under the assumption of a white excitation noise. Such a pole can be eliminated provided that an a priori information is available. This is the case of the harmonics of the rotation speed for rotating machines with load unbalancing. Similarly, a colored excitation noise can easily be eliminated provided that its poles are (significantly) more damped than those of the structure. It is also a common finding that the poles of the structure tend to stabilize when the Hankel matrix order is increased, whereas those of the excitation mostly do not. A nonstationary excitation is actually a favorable situation, since the averaging performed in (24) for computing $G$ tend to whiten the noise. This is the case of a chirp-like nonstationary excitation.

$\mathrm{RR} \mathrm{n}^{\circ} 4024$ 


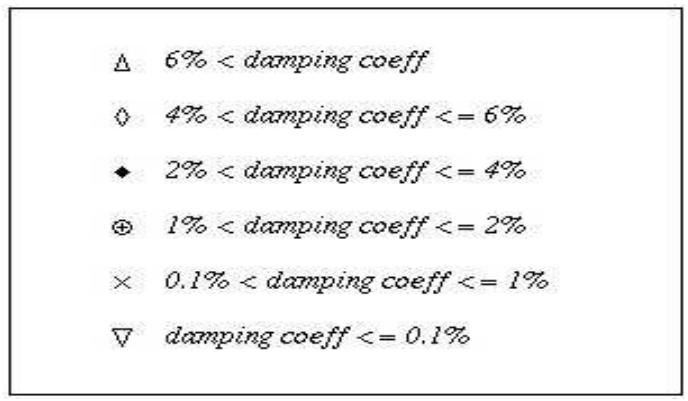

Figure 1: Meaning of the symbols in Fig. 2 to 4.

Real application example The proposed method has been applied to the Swiss Z24 bridge, a benchmark of the BRITE/EURAM project SIMCES on identification and monitoring of civil engineering structures, for which EMPA (the Swiss Federal Laboratory for Materials Testing and Research) has carried out tests and data recording. The response of the bridge to traffic excitation under the bridge has been measured in 139 points, mainly in the vertical and transverse directions, and sampled at $100 \mathrm{~Hz}$. Because at most 19 sensors were available, 9 data sets have been recorded, each containing the measurements from 4 fixed and 15 moving sensors. However, it is not recommended to merge data recorded under too different temperature and traffic conditions [9], and to mix different directions [10]. Therefore, for investigating the experimental properties of the proposed polyreference subspace algorithm. We have selected $J=2$ records from sensors in the vertical direction, with one reference sensor, and two moving sensors in the first record, and only one moving sensor in the second record. Each signal contains 65535 samples. As mentioned above, processing more records would have affected the required memory size, but not the computational complexity. Also, it is of interest to investigate the practical relevance of the conceptual capability of the proposed algorithm to handle records with different numbers of moving sensors.

The classical subspace algorithm of section 2, based on full data covariances and on $q=128$, has been applied separately to each record, which contain 3 and 2 sensors, respectively. The results are displayed in Fig. 2 and 3, where frequencies (ordinate) are displayed for increasing SVD truncation order (abscissa), in a stabilization diagram which symbols are explained in Fig. 1. Typical values of estimated modes which can be extracted from these diagrams are displayed in Table 1. 
Table 1: Estimated modes - Classical subspace identification.

\begin{tabular}{|c|c|c|c|c|c|}
\hline Mode & 1 & 2 & 3 & 4 & 5 \\
\hline Frequency $(\mathrm{Hz})$ & 4 & 5.3 & 9.8 & 10.3 & 12 \\
\hline
\end{tabular}

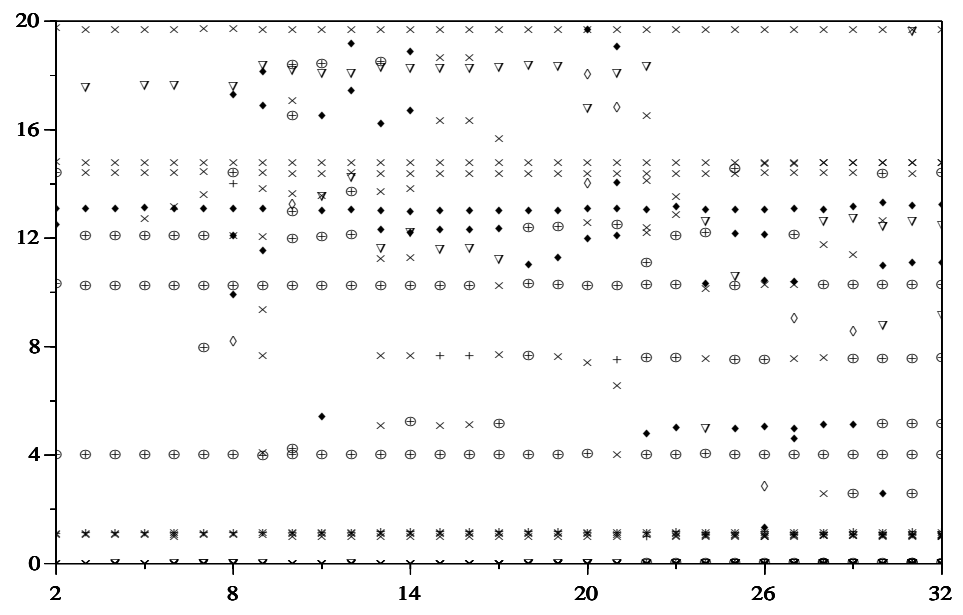

Figure 2: Classical subspace identification: first record (3 sensors).

$\mathrm{RR} \mathrm{n}^{\circ} 4024$ 


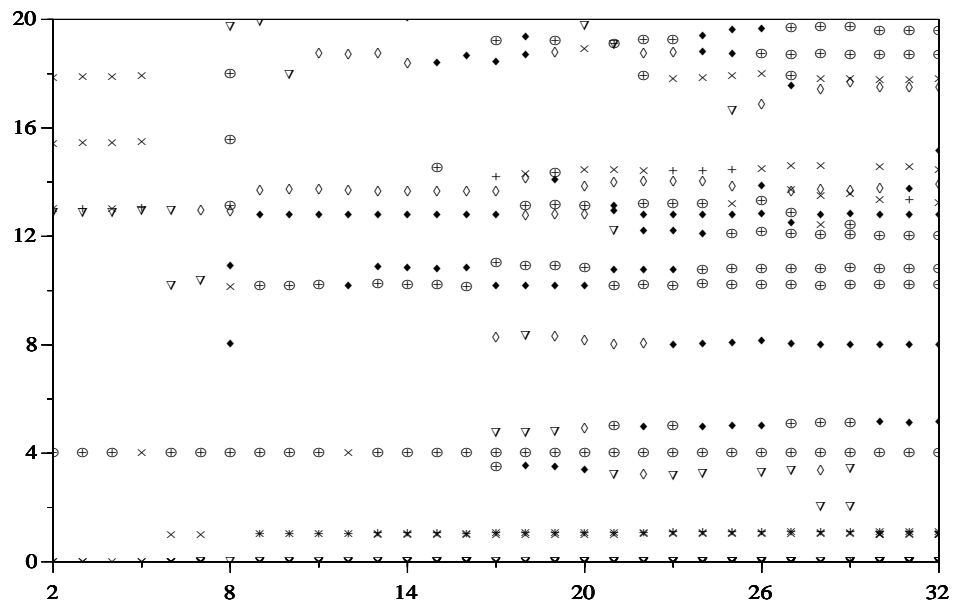

Figure 3: Classical subspace identification: second record (2 sensors).

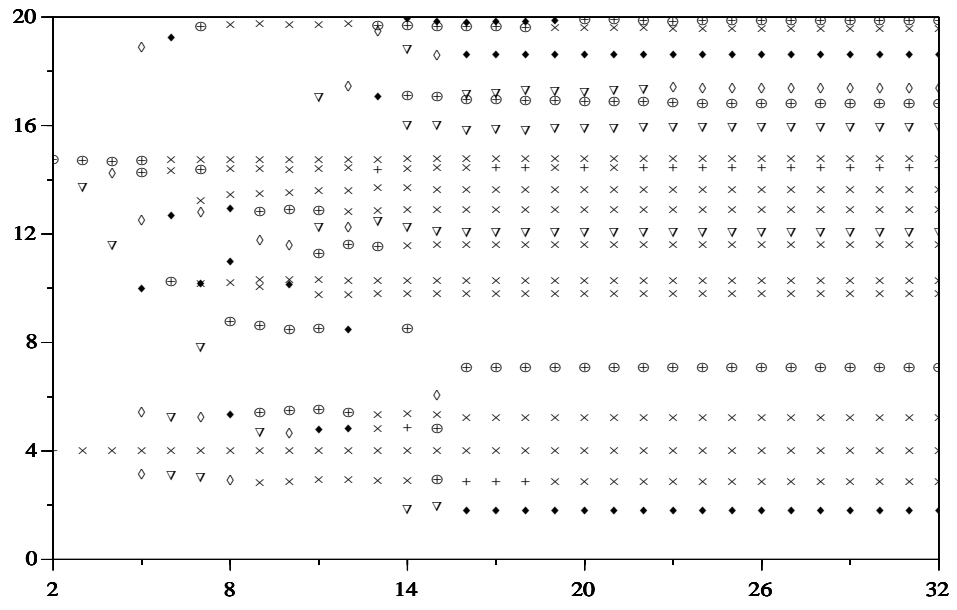

Figure 4: Polyreference subspace identification with the two records. 
Polyreference subspace eigenstructure identification The polyreference subspace identification algorithm of section 4 , based on partial covariances ${ }^{1}$ (8) and on $q=128$, has been applied to the data from the two records. The results are displayed in Fig. 4. Since the Hankel matrix $\overline{\mathcal{H}}$ is built on stacked covariances (22), its SVD truncation order should be taken no greater than $128 \times 1 /(1+2+1)=32$. Fig. 4 shows up several improvements over Fig. 2 and Fig. 3, on several aspects. Actually, for large enough truncation order:

- Spurious modes disappear: around $1 \mathrm{~Hz}$, for example;

- True modes appear and stabilize faster and fluctuate less in the diagrams: around $5.3 \mathrm{~Hz}$ and $9.8 \mathrm{~Hz}$, for example;

- The damping of the true modes is more acurately estimated, at lower orders than before, allowing to separate true and wrong modes among the stabilized ones: a wrong mode appears at $7 \mathrm{~Hz}$ with a high damping, whereas the true mode at $4 \mathrm{~Hz}$ appears with a low damping;

- Some modes, not identified on Fig. 2 and 3, now show up in a stable diagram: around $12 \mathrm{~Hz}$, among others.

The polyreference method extracts 7 modes in the range $0-11 \mathrm{~Hz}$. Five of these modes come from the structure and tend to appear in many diagrams during the whole year. The $5.3 \mathrm{~Hz}$ mode is very difficult to extract, due mainly to low excitation for this frequency. In both classical identifications, it appears very late in the diagrams and has little stability, whereas those two issues are overcome in the polyreference trial. Fig. 4 is a remarkably clear diagram which, above a truncation order of 15 , is cleaned from all spurious poles, even those which tend to stabilize in Fig. 2 and 3.

What the proposed algorithm is not capable of is to separate modes of the structure from the modes corresponding to the non-white excitation, but the classical subspace identification procedure is unable to achieve that either. Nevertheless, the new algorithm appears to perform well at extracting modes which appear only in some diagrams, for example modes which are not very well excited all the time. This feature is important in practice, and should hopefully be confirmed in further numerical investigations.

\footnotetext{
${ }^{1}$ Note that these covariance matrices are column vectors, since only one reference sensor is available in each of the two records.
}

$\mathrm{RR} \mathrm{n}^{\circ} 4024$ 


\section{Conclusion}

We have discussed the polyreference setup for blind eigenstructure identification. This type of setup is popular for instance in vibration mechanics, where several moving sensors mimic the availability of a much larger set of sensors. The proposed identification method consists in merging the data first and processing them globally using an output-only covariance-based subspace algorithm. This has to be contrasted with classical polyreference identification, which is usually performed by merging identification results obtained on the different records. The presence of an unmeasured and nonstationary excitation makes the issue of output data merging nontrivial. The key idea of the proposed method is a suitable normalization of the output covariances. This method has been proved to provide us with consistent estimates of the eigenstructure, even under nonstationary excitation. Some numerical results on one real example have been reported, which highlight the potential benefits of the proposed method. First, the method efficiently merges data by smoothing out the diagrams, rejecting spurious and instable frequencies. Second, applying the polyreference method over time is expected to smooth out further the effect of nonstationary excitation. Finally, the actual stability of the polyreference diagrams makes the proposed polyreference method a good candidate for a future automated identification procedure. 


\section{References}

[1] M. Abdelghani, M. Goursat, T. Biolchini, L. Hermans and H. Van der Auweraer, "Performance of output-only identification algorithms for modal analysis of aircraft structures", IMAC-XVII, Int. Modal Analysis Conf., Kissimmee, FL, Feb.1999.

[2] M. Basseville, M. Abdelghani and A. Benveniste, "Subspace-based fault detection algorithms for vibration monitoring", Automatica, vol.36, no 1, pp.101-109, 2000 .

[3] M. Basseville, A. Benveniste, B. Gach-Devauchelle, M. Goursat, D. Bonnecase, P. Dorey, M. Prevosto and M. Olagnon, "Damage monitoring in vibration mechanics: issues in diagnostics and predictive maintenance", Mech. Syst. Sign. Proc., vol.7, n.5, pp.401-423, 1993.

[4] A. Benveniste and J-J. Fuchs, "Single sample modal identification of a nonstationary stochastic process", IEEE Trans. Automat. Contr., vol.30, n.1, pp.66-74, 1985.

[5] F. Deblauwe, R.J. Allemang and D.L. Brown, "The polyreference time domain technique", IMAC-V, Int. Modal Analysis Conf., pp. 832-845, 1987.

[6] L. Hermans and H. van der Auweraer, "Modal testing and analysis of structures under operational conditions: industrial applications", Mech. Syst. Sign. Proc., vol.13, n.2, pp.193-216, 1999.

[7] B. Ottersten, M. Viberg and T. Kailath, "Analysis of subspace fitting and ML techniques for parameter estimation", IEEE Trans. Sign. Proc., vol.40, pp.590599, 1992.

[8] B. Peeters and G. de Roeck, "Reference-based stochastic subspace identification for output-only modal analysis", Mech. Syst. Sign. Proc., vol.13, n.6, pp.855878.

[9] B. Peeters and G. de Roeck, "One year monitoring of the Z24-bridge: environmental effects versus damage events", IMAC-XVIII, Int. Modal Analysis Conf., San Antonio, TX, Feb.2000.

[10] M. Prevosto, M. Olagnon, A. Benveniste, M. Basseville, G. Le Vey, "State-space formulation, a solution to modal parameter estimation", Jal Sound and Vibr., vol.148, n.2, pp.329-342, 1991. 
[11] P. Stoïca and R.L. Moses, Introduction to Spectral Analysis, Prentice Hall, 1997.

[12] P. Van Overschee and B. De Moor, Subspace Identification for Linear Systems: Theory, Implementation, Applications, Kluwer, 1996.

[13] M. Viberg and B. Ottersten, "Sensor array processing based on subspace fitting", IEEE Trans. Sign. Proc., vol.39, no 5, pp.1110-1121, 1991. 


\section{Contents}

1 Introduction $\quad 3$

2 Output-only covariance-driven subspace identification 4

3 Polyreference subspace identification: stationary excitation 6

4 Nonstationary excitation: data normalization $\quad 8$

5 Nonstationary excitation within the records $\quad 11$

6 Structural vibration analysis example $\quad \mathbf{1 4}$

6.1 Modeling . . . . . . . . . . . . . . . . . . 15

6.2 Numerical results . . . . . . . . . . . . . . . . . . . 16

7 Conclusion $\quad 22$

$\begin{array}{ll}\text { References } & \mathbf{2 3}\end{array}$ 


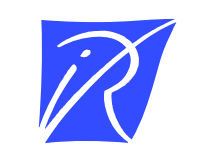

Unité de recherche INRIA Lorraine, Technopôle de Nancy-Brabois, Campus scientifique, 615 rue du Jardin Botanique, BP 101, 54600 VILLERS LÈS NANCY

Unité de recherche INRIA Rennes, Irisa, Campus universitaire de Beaulieu, 35042 RENNES Cedex

Unité de recherche INRIA Rhône-Alpes, 655, avenue de l'Europe, 38330 MONTBONNOT ST MARTIN

Unité de recherche INRIA Rocquencourt, Domaine de Voluceau, Rocquencourt, BP 105, 78153 LE CHESNAY Cedex

Unité de recherche INRIA Sophia-Antipolis, 2004 route des Lucioles, BP 93, 06902 SOPHIA-ANTIPOLIS Cedex

Éditeur

INRIA, Domaine de Voluceau, Rocquencourt, BP 105, 78153 LE CHESNAY Cedex (France)

http://www.inria.fr

ISSN 0249-6399 